\title{
Some Problems in Application of Integrated Radiation Thermopile Sensor
}

\author{
by A. Marincic ${ }^{1}$ and R. Tmusic ${ }^{2}$
}

${ }^{1}$ Faculty of Electrical Engineering, University of Belgrade, Bulevar Revolucije 73, 11000 Belgrade, Yugoslavia, E-mail: emarinci@ubbg. etf.bg.ac.yu

${ }^{2}$ Delyug, Treci bulevar 23/1 11000 Belgrade, Yugosavia, E-mail:Iune@EUnet.yu

\begin{abstract}
In this paper some problems that arise in application of integrated radiation thermopile sensor Heimann TPS 535, incorporated in module TPMF2, in application that measures temperature of movable heating plates, that are used in food processing industry, were investigated. Series of experiments of cooling of a previously heated body were performed and linearization of integrated radiation thermopile sensor output voltage was accomplished within $\pm 1.5^{\circ} \mathrm{C}$. Thermographic verification was carried out, in order to optimize characteristics of described system application.
\end{abstract}

\section{Introduction}

The most common type of low-temperature radiation thermometers, used in day to day industrial applications is based on blackened thermopile as a detector. In these thermometers radiation is focused by the means of lenses or mirrors on the measuring junction of thermocouples, while cold junctions are shielded, and therefore, have practically the environment temperature. Advanced integration technology in manufacturing sensors whose thermopile is placed on a thin isolating membrane $(\alpha \sim 1 \mu \mathrm{m})$, significantly improve their sensitivity due to larger thermal resistance [1]. Various materials could be used for thermoelements. In recent sensors, Si-Al thermoelements are being employed, mainly because they have linear characteristic and fairly good sensitivity, use standard technology and have possibility of incorporating the signal conditioning electronics in the sensor chip [2] (Fig 1).

Manufacturers of instruments that measure temperature employing detectors of this type emphasize emissivity as a possible cause for readout error, and propose two methods that should help in overcoming the problem: in situ Calibration, with appropriate contact sensor, or use of emissivity charts, mostly only in cases when direct contact is not possible. The facts that in most cases other factors than emissivity have minor influence on readout, and that it is actually impossible to distinguish different contribution to the energy loss between source and detector favor the practice that correction of readout on all commercial instruments is called emissivity correction. In this way, all other factors are considered of minor importance, but for accurate measurements it is very often not so. Most instruments use fixed focus optical system that causes readout to drop with distance from the target, although the target is still in the full field of view of the detector.

Apart from problems that can be designated as static, in cases where radiation thermometer is used for temperature measurement on movable objects, problems arise due to the finite response speed of particular detector, which can be designated as dynamic. And, at the end, very serious problem that causes systematic errors that are hard to avoid comes from the non-uniformity of thermal field of an object, especially in cases of measurement on objects that come in series, in regular time intervals. In such cases, if we presume that objects are identical, it is important to know at which moment samples will be taken.

\section{Experimental results}

Being an instrument that is used in comparatively low temperature range, the radiation thermometer incorporating this kind of sensor must work on a wide band basis. This 
principle, however, has some disadvantages, the emissivity dependence of instrument readings being one of them that in practice require calibration in every particular case. For this reason, a practical method of in situ calibration has been developed, with the purpose of adjusting instrument to every particular application.

In applying this method of operation, calibration and operation regimes are used. It is also required to eliminate noise by filtration, to make emissivity correction, correction to the distance, and sampling or duty cycle correction.

With assumption that time constant of sensor is fairly shorter than the time constant of heating plate, calibration in quasi stationary regime was performed, during the self-cooling process of heating plate. Self-cooling was chosen, as this process has much better uniformity of surface temperature, than heating process, especially when concentrated energy from electric heating element is applied. To verify this assumption, thermographic record of heating plate, both in heating and self-cooling regime was taken. (Fig. 2, 3).

It was found that calibration curves can be approximated parabolically, giving readout error under $\pm 1,5^{\circ} \mathrm{C}$. Representative calibration error curves obtained for different maximal temperature are given in fig.4. Greater error values at the beginning of the cooling process correspond with unevenness of the surface temperature that quickly disappears when cooling takes place. In this way, it is possible to establish calibration curve for every heating element- detector combination, in a short time.

Another error, which requires a careful investigation, is change of thermometer readout with target distance. The investigated thermopile sensor module Heimann TPMF 2 showed significant change oh temperature readout even within the range in which the target is still in

Change of temperature readout with target distance, for an instrument made with Heimann TPMF2 module, is shown on fig.5. To illustrate importance of optical system the full field of view of the sensor.quality, same curve is taken for an instrument with a better optical system, Uitrakust R20 commercial model (Fig 6).

It should be noted, however, that this correction is not necessary in many industrial applications where target distance is not likely to be changed during measurement, but in such cases it is good to place detector in position where small distance changes will have the smallest effect. From shapes of series of output voltage curves vs. distance for different starting temperatures it was demonstrated that such a specific distance exists. Then, from $\mathrm{dU} / \mathrm{dx}_{\mathrm{t}}$ shown on the fig. 7 , it is possible to draw conclusion that the best position for Heimann TPMF2 module is $225 \mathrm{~mm}$ from the target.

In food processing industry, one of the most important parameters is temperature of the heater applied to raw material. From fig 2, however, it is possible to see that considerable differences in instrument readout will arise, according to the position of the sensor respectively to the heating plate. For this purpose, series of measurements were made with calibrated instrument, in order to determine change in temperature readout, according to instrument position.

Results of these measurements are given in fig. 8. For successful contactless temperature measurement it is necessary to keep object fully in the instrument field of view. From fig. 8, however a conclusion can be drawn, that even with this condition fulfilled, measurement result can vary greatly with instrument position. Best results in every particular case are thus obtained by comparative analysis of results from thermogram and radiation sensor, in order to determine position of sensor that gives the most representative result, with a minimum error.

In one of our experiments, sensor was used for temperature measurement on heater plates that were moving with some constant speed in the instrument field of view. Measurement method used pitch extraction from input signal, in order to take sample at the same point of every plate. It is essential for this method that the object moves with such speed that it is at least $5 \tau$ ( $\tau$ being the time constant of sensor) in the field of view of the instrument. Noise filtration, however, has impact to response time, and it is advisable to take care not to overfilter input signal, if fast response is desirable. On the other hand, in order to obtain uniform readout, if objects are moving in front of the detector too fast respectively to 
its response time, it is desirable to filter signal more. In such cases, if objects pass with some empty space between them (duty cycle $\mathrm{C}<1$ ), readout has to be corrected respectively to duty cycle and background temperature as follows:

$$
U_{\left(\tau_{v}\right)}=\frac{1}{C} U_{u f f}-\frac{1-C}{C} U_{b}
$$

where $U_{\left(T_{0}\right)}$ stands for output voltage that would be produced by object at temperature $T_{0}$ $U_{\text {eff }}$ for actual output voltage, $C$ for duty cycle, and $U_{b}$ for the voltage that is produced by background radiation. If background radiation is constant, it is possible to replace $\frac{1-C}{C} U_{h}$ with a constant for that background temperature.

Emissivity correction is, in a way, avoided when in situ calibration is performed, as it is already taken into account during calibration. It is possible to perform additional (mathematical) correction of the emissivity, but it means that it is necessary to know its exact value during calibration, as well as exact new value, which, in reality makes it more simple to recalibrate instrument if different emissivity value than the one during calibration is suspected.

\section{Conclusion}

It was shown that software linearization of sensor output can be achieved within $\pm 1.5^{\circ} \mathrm{C}$, by parabolic approximation, in fixed geometry, i.e. if strict control of the target distance and angle is performed. In such cases, when no change in emissivity and geometry of the system is expected for long time, the most convenient way to measure temperature is to calibrate the system in situ. Apart from that, it is necessary to perform analysis of temperature field on the object surface, in order to get best sensor position, especially if heating is performed by means of concentrated source, i.e. electric heating element. Since radiation thermometers are often used for temperature measurement of moving objects, it is important to take into consideration effects of finite response time of detector, and to use different approach in cases when speed of the object overcomes possibilities of detector. Because of the simple linearization, low price, fast response, long-term stability and large sensitivity in the lowtemperature range, the investigated sensor, in our opinion, is very convenient for a variety of industrial applications, provided that measurement system takes into consideration all described limitations. It is worth of emphasizing, however, that measurement with this type of instrument requires more precaution than necessary when using thermal imaging camera, where object emissivity is practically only problem that has to be considered

\section{REFERENCES}

[15] GARDNER (J.) - Microsensors -, Principles and Applications, John Wiley \& Sons, Inc., New York, 1994.

[16] SZE (S. M.), Editor, - Semiconductor Sẹnsors, A Wiley-Interscience Publication, John Wiley \& Sons, Inc., New York, 1994.

[17] SCHIEFERDECKER (J.), SCHMIDT (W) Thermal Sensing Devices Infrared sensors, EG\&G Heimann, Application Notes, TPS Bulletin 5/94:

[18] TMUSIC (R.), M. Sc. thesis Faculty of Electrical Engineering,Belgrade University, 1997.

E. O. Doebelin, Measurement systems, MoGraw-Hill Book Co., London, 1991.

[19] Guide to the expression of uncertainty in measurement, BIPM, Paris, 1993. 


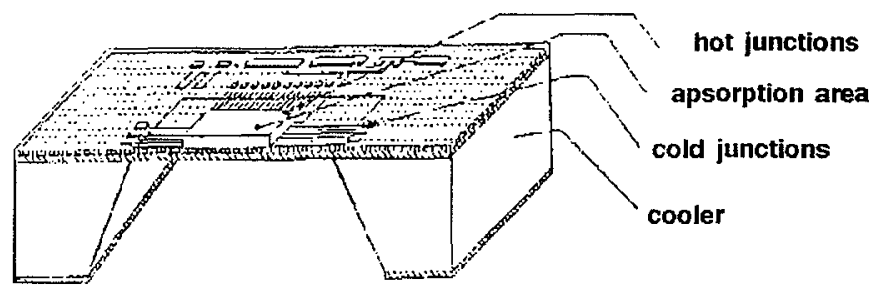

Fig. 1. Simplified outlook of integrated thermopile sensor

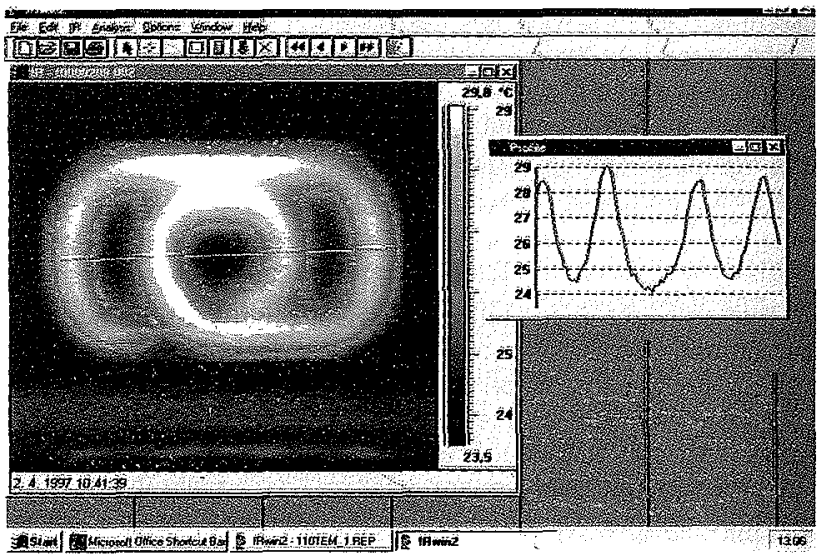

Fig. 2. Thermogram of heating of electrically heated plate. Curve on the right side represents temperature change along horizontal axis (the white line on the thermogram).

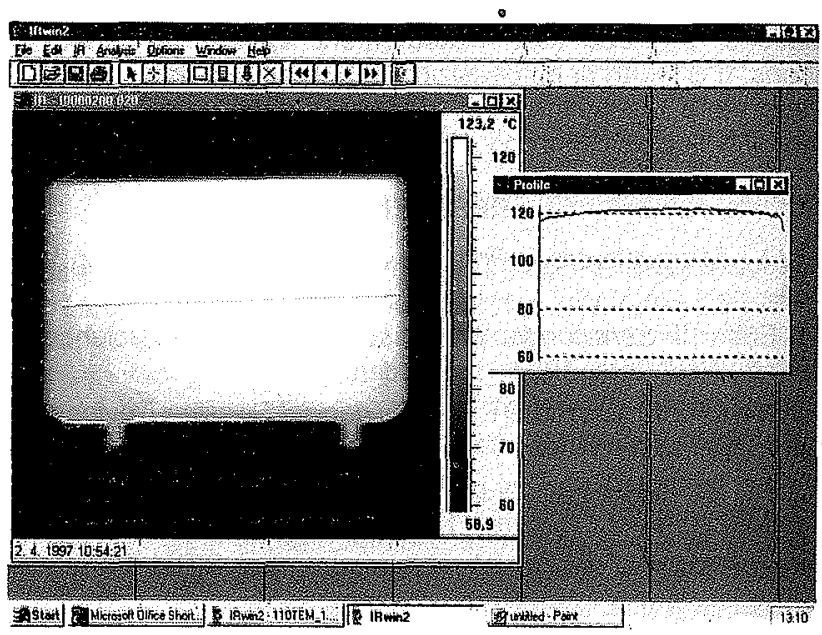

Fig. 3. Thermogram of cooling of heated plate from Fig. 2. Zurve on the right hand side clearly shows better uniformity of surface temperature, than for an object heated with concentrated source of energy. 
http://dx.doi.org/10.21611/qirt.1998.047

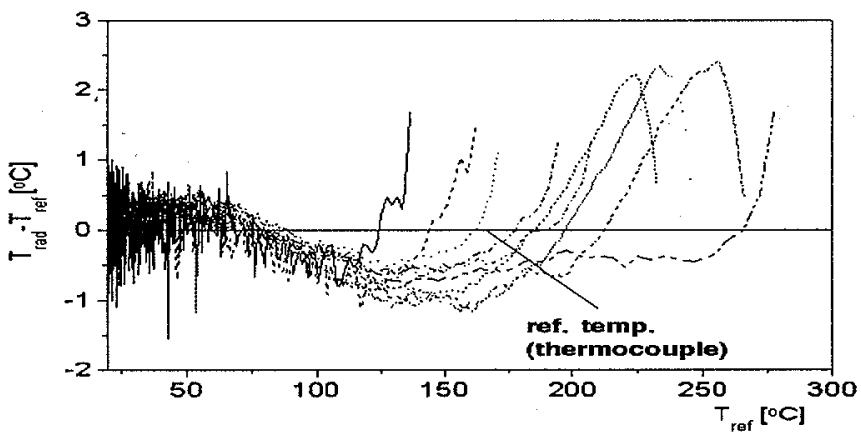

Fig. 4. Calibration error vs. temperature, for different starting temperatures. Greater error values at the beginning of calibration process are due to the fact that object was heated with concentrated source of energy.

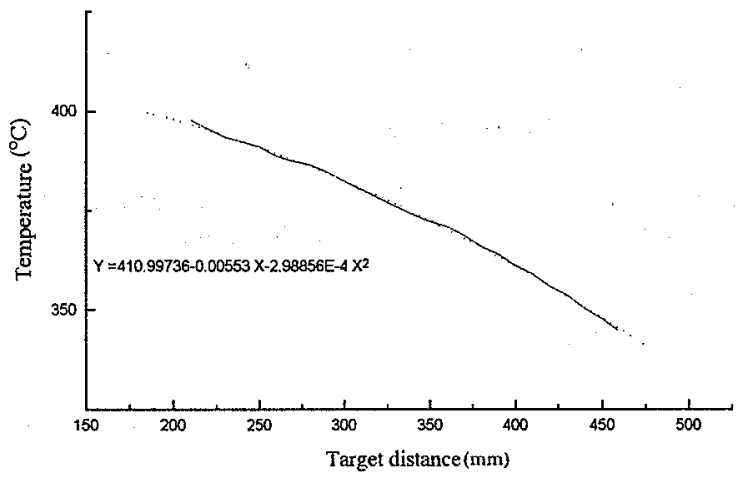

Fig. 5. Change of thermometer readout with target distance (Heimann TPMF2)

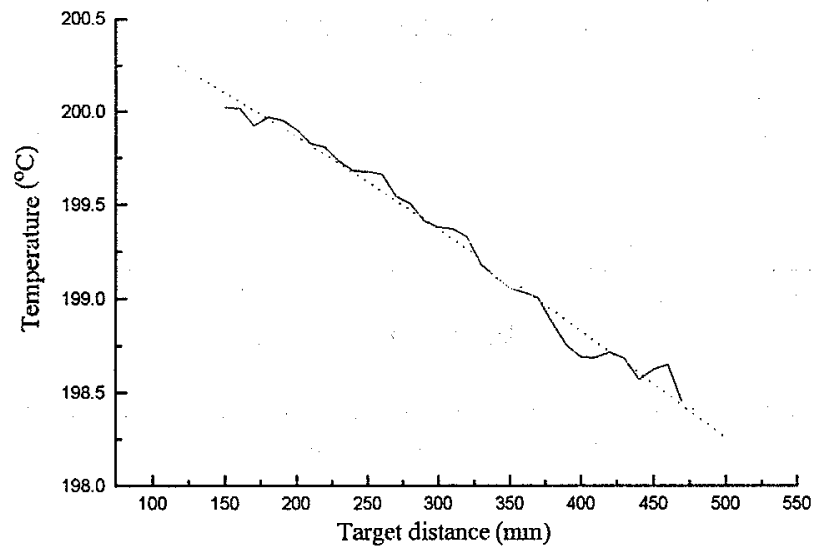

Fig. 6. Change of thermometer readout with target distance (Ultrakust R20) 
http://dx.doi.org/10.21611/qirt.1998.047

Absolute value of $d U / d x$

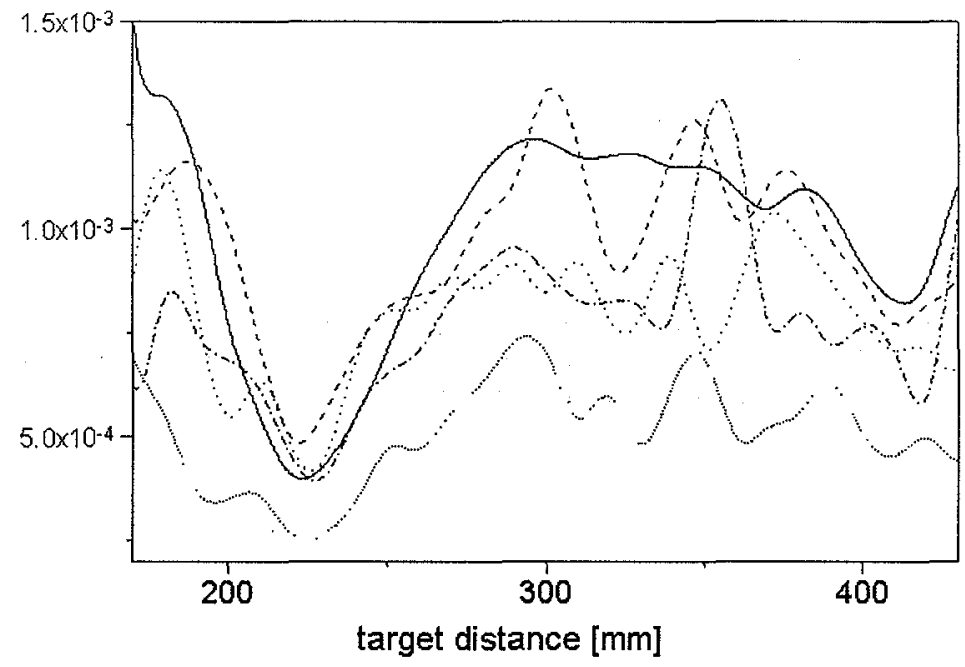

Fig. 7. Output voltage derivative with distance to the target (dU/dx) for Heiman TPMF2 sensor for different starting temperatures

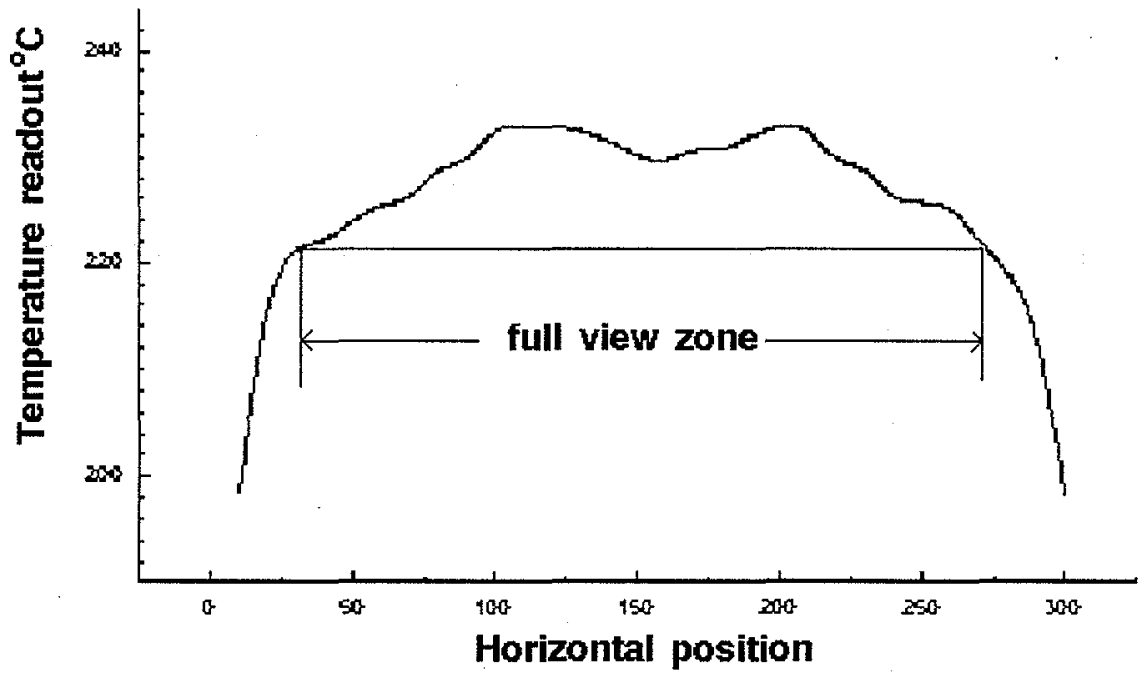

Fig. 8. Surface temperature change vs. sensor horizontal position, distance to the target being constantly $300 \mathrm{~mm}$ 